\title{
BIRTH DEFECTS AND CHILDHOOD CANCER: A SHARED BIOLOGICAL PATHWAY FOR HIRSCHSPRUNG DISEASE AND HEPATOBLASTOMA DEVELOPMENT?
}

\author{
Talita Aguiar*1, Anne Caroline Teixeira ${ }^{1}$, Juliana Sobral ${ }^{1}$, Silvia Souza Costa ${ }^{1}$, Marília \\ Scliar $^{2}$, Renata Hall-Nielsen ${ }^{3}$, Ana Regina Ramos ${ }^{4}$, Beatriz dos Santos ${ }^{4}$, Valentina \\ Oliveira $^{4}$, Ariane Backes ${ }^{4}$, Osvaldo Artigalás ${ }^{4}$, Isabela Cunha ${ }^{5}$, Carla Rosenberg ${ }^{1}$, Eugênia \\ Valadares $^{3}$, Raquel Pinto ${ }^{4}$, and Ana Krepischi ${ }^{1}$ \\ ${ }^{1}$ Institute of Biosciences, University of Sao Paulo \\ ${ }^{2}$ University of São Paulo \\ ${ }^{3}$ Benjamim Guimarães Foundation \\ ${ }^{4}$ Grupo Hospitalar Conceição \\ ${ }^{5}$ Rede D'OR-São Luiz
}

July 1, 2020

\begin{abstract}
Background: The association of birth defects and developmental alterations with pediatric cancer is a recognized long-standing global observation, although very few syndromes confer a high risk of hepatoblastoma development. Procedures: Here we describe the results of germline exome analysis of two syndromic patients with Hirschsprung disease who developed hepatoblastoma. Results: Germline variants of uncertain clinical significance (VUS) were disclosed in 28 genes that might be related to patients' phenotypes. More importantly, germline VUS were detected in eight known cancer predisposition genes (APC, BRCA1, ERBB2, ERCC2, HRAS, ODC1, SERPINA6, and MCC). Additionally, our data disclosed two candidate genes with germline variants potentially contributing to the phenotype of these patients, namely, CEP164 and CYP1A1. The last one was the only gene presenting variants of uncertain significance in both patients. This gene encodes the most important xenobiotic-metabolizing enzyme of the placenta for which relevant inducible activity has been demonstrated throughout pregnancy. Conclusion: our data pointed out a set of genes that are enriched for pathways already related to cancer and developmental biology, suggesting they could have a broader role in cancer and congenital abnormalities. These results can help future studies to understand the biology of Hirschsprung disease and its association with hepatoblastoma.
\end{abstract}

\section{INTRODUCTION}

The etiology of pediatric cancer is largely unknown ${ }^{1}$. Recent studies of large cohorts of pediatric cancer have shown that approximately $10 \%$ of the patients carry germline pathogenic variants in a broad spectrum of cancer susceptibility genes ${ }^{2-5}$. The evidence of the link between pediatric cancer and congenital anomalies is robust ${ }^{6-8}$, however, the etiology of most of these associations remains underexplored.

Hepatoblastoma (HB) is the most common malignant tumor of liver in the pediatric population ${ }^{9}$, although it is considered an ultrarare disease, accounting for only $1 \%$ of pediatric tumors ${ }^{10,11}$. In Brazil, collected data on hepatoblastomas are concordant with the worldwide prevalence ${ }^{12-14}$. Increased risk for hepatoblastoma development has been reported in association with specific congenital syndromes, including Beckwith-Wiedemann ${ }^{15,16}$, familial adenomatous polyposis (FAP) ${ }^{17}$, and trisomy of chromosome $18^{18}$. Non-genetic factors known to be associated with hepatoblastoma risk are mainly related to very low birth 
weight $(<1500 \mathrm{~g})$, including preterm birth $(<33$ weeks), small size for gestational age, and multiple birth pregnancies $^{9,19}$. To date, there is a single report of a patient with hepatoblastoma and Hirschsprung disease 20 , which is a rare congenital anomaly resulting from the absence of enteric neurons at the end of the bowel, affecting about 1-5 in 10,000 newborns ${ }^{21,22}$. Related symptoms arise because there is no propulsive motility in the aganglionic bowel as a consequence of defective neural crest cell development, causing severe chronic constipation, abdominal distension, vomiting, and growth failure ${ }^{22,23}$. Hirschsprung disease is a multigenic disorder with variable penetrance and severity, characterized by extensive genetic heterogeneity. Predisposing mutations have been recognized in several genes, including RET, GDNF, GFRA1, NRTN, EDNRB, EDN3, ZEB2, PHOX2B, SOX10, SHH, ECE1, DHCR7, L1CAM, KIF1BP, BBS1-BBS11 and RMRP ${ }^{22,24 .}$ Some conditions, such as chromosome 21 trisomy, increase the risk for Hirschsprung disease ${ }^{25}$. The sibling recurrence rate varies according to both the length of the aganglionic bowel and to the sex of the first affected sibling ${ }^{26,27}$ in a pattern of multifactorial inheritance with different liability threshold for sex.

In the course of genomic studies in a cohort of Brazilian individuals with childhood cancer, we ascertained one additional syndromic patient presenting Hirschsprung disease and hepatoblastoma, besides the first case reported by Pinto et al. (2016) ${ }^{20}$. In this report, we performed a germline exome analysis of these two patients in whom the association of Hirschsprung disease and hepatoblastomas was documented, and of one parent of each patient. We also investigated the spectrum of somatic mutations in one of the tumors.

\section{METHODOLOGY}

\section{DNA samples}

Written informed consent was obtained from the parents of the two studied patients. Patient 1 was referred from Department of Pediatrics Hospital da Baleia - Brazil, and Patient 2 was referred from Hospital da Criança Conceição - Brazil.

Genomic DNA samples were extracted from peripheral blood of both patients and their available parents (mother of Patient 1 and father of Patient 2), and formalin-fixed paraffin-embedded (FFPE) tumor tissue (biopsy) from Patient 1. DNA from peripheral blood was isolated using phenol-chloroform extraction followed by Ethanol precipitation ${ }^{28}$. DNA from FFPE tumor sample was obtained in the AC Camargo Tumor Bank 29; tissue embedded in paraffin, direct cut $(10 \mu \mathrm{g})$ and phenol-chloroform extraction were used.

\section{Library preparation and sequencing}

Genomic libraries of whole-exome were constructed using $1 \mathrm{ug}$ of genomic DNA and the Sureselect QXT V6 (Agilent Technologies - Patients 1 and 2, and Patient 1's mother), OneSeq Constitutional Research Panel (Agilent Technologies - Patient 2's father), and xGen Exome Research Panel v1.0 (IDT - Integrated DNA Technologies - FFPE tumor sample) kits. Enriched libraries were sequenced on the Illumina HiSeq 2500 platform in paired end reads. The sequences were aligned to the GRCh37/hg19 human genome reference with the BWA_MEM algorithm ${ }^{30}$. Picard tools (v.1.8, http://broadinstitute.github.io/picard/) were used to convert the SAM file into BAM and to mark PCR duplicates. The Genome Analysis Toolkit (GATK 3.7) 31 were used to realign indels, recalibrate the bases, and to call (Unified Genotyper) and recalibrate variants (VQSR). Finally, multiallelic variants were split into different lines using the script split_multiallelic_rows.rb from Atlas2 ${ }^{32}$ to obtain the VCF files used for analysis.

\section{WES data analysis}

We used VarSeq software version 1.5.0 (Golden Helix) to annotate and filter the variants. SNV and indel variants were filtered by read depth $(>10)$, Phred score $(>20)$, and variant allele frequency $(>0.35$ for germline, and $>0.10$ for somatic variants). Variant annotation was performed based on public databases of populational, clinical, and functional databases.

Germline variants with populational frequencies above $0.5 \%$ or $1 \%$ for recessive and dominant models of inheritance, respectively, were filtered out. The somatic mutations in the tumor sample were obtained excluding all germline variants. Following, variants were filtered based on Sequence Ontology by RefSeq, and 
only coding non-synonymous missense and essential splice site, frameshift, and gain/loss of stop-codons (loss of function - LoF) were maintained for further analysis. In silico prediction of pathogenicity of missense variants were based on six algorithms provided by the database dbNSFP (version 2.4). The potential damaging effect was also assessed using the VEP32 script software package from Ensembl (https://www.ensembl.org/), and only variants predicted as pathogenic by at least five different tools were prioritized. All the LoF variants were also prioritized. The final list of filtered variants was annotated using Varelect ${ }^{33}$ and HPO ${ }^{34}$ for ranking genes associated to the specific phenotype of the patients. The variants were validated by visual inspection using the Integrated Genomics Viewer (IGV). The prioritized germline variants were classified according to the ACMG guidelines ${ }^{35,36}$, using the Varsome tool ${ }^{37}$. The Supporting Information Figure S1 summarizes the approach for WES data analysis.

Two prioritized germline variants from candidate genes (CYP1A1 and CEP164) and mutational hotspots of TERT promoter were investigated by Sanger sequencing (primer pairs are available under request).

\section{Copy number alterations (CNAs)}

Germline chromosome microarray analysis was performed for Patients 1 and 2 using a 180K platform (Agilent Technologies), as previously reported ${ }^{38}$. Somatic CNA events in the tumor from Patient 1 were derived from exome data obtained from the FFPE sample using the software Nexus Copy Number 9 (Biodiscovery), with the SNP-FASST2 segmentation algorithm (threshold $\log _{2}$ Cy3/Cy5 ratio of $|0.2|$ for gains and losses; $|1.2|$ for high copy number gains and homozygous losses; minimum LOH length of $10 \mathrm{Mb}$ ). Common CNVs (Database of Genomic Variants, http://dgv.tcag.ca/dgv/app/home) were disregarded.

\section{PATIENTS'S MEDICAL REPORTS}

Patient 1 is a female, third child of non-consanguineous parents, born at term by cesarean section; her two siblings have a normal phenotype. Abdominal distension and no evacuation were detected in the first 24 hours after birth, and the diagnosis of Hirschsprung disease was made. In the clinical evaluation at 5 years old, she presented with global neuropsychomotor delay, facial dysmorphisms, nail dysplasia (hypoplastic), and clinodactyly. Hepatoblastoma was diagnosed at the age of four, being classified as Epithelial Subtype with a predominance of embryonal cells, PRETEXT IV, high-risk. The patient was submitted to the chemotherapy protocol SIOPEL6. She died before the surgical procedure.

Patient 2 is a male, third child of a consanguineous couple; his sister was born with congenital bilateral cataract, while his brother exhibited intestinal atresia-terminal ileus. The patient was born premature (28 weeks) and his mother, who presented gestational risk (cardiac defect and preeclampsia), died during his birth due to congestive heart failure. At birth, the patient underwent mechanical ventilation and was admitted to the intensive care unit for periventricular encephalomalacia, grade II intracranial hemorrhage on the right, seizures and sepsis. He was born with a syndromic phenotype, composed of congenital ileal atresia, bilateral cataract and sensorineural deafness. In the first 48 hours of age, the patient developed abdominal distension and vomiting. During laparotomy, intestinal atresia in the terminal ileum and a disconnected cecum were identified. Histopathological examination also revealed absence of ganglion cells in the rectum and sigmoid colon, consistent with Hirschsprung disease that was corrected surgically. The hepatoblastoma tumor was diagnosed at 25 months of age, classified as Fetal Epithelial Subtype, PRETEXT II, low risk. He was submitted to the chemotherapy protocol with cisplatin, doxorubicin and ifosfamide, followed by partial hepatectomy. Currently, the patient is in post-treatment follow-up. Further clinical details were published by Pinto et al. (2016) ${ }^{20}$.

\section{RESULTS}

\section{Germline variants}

Chromosome microarray analysis excluded the presence of pathogenic germline CNVs in both patients. The Patient 2 carries an interrupted duplication encompassing a total segment of $\sim 364 \mathrm{~kb}$ at $14 \mathrm{q} 23.2$, not inherited from his father (arr[GRCh37] 14q23.2(64374657_64435014)x3; $\operatorname{arr}$ [GRCh37] 14q23.2(64474837_64738458)x3; Figure 1); maternal material was not available for segregation analysis. This CNV partially duplicates the 
genomic sequence of two Morbid OMIM genes (SYNE2 \#608442 andESR2 \#601663) and was classified as VUS. Rare duplications overlapping this region were reported in the Database of Genomic Variants (DGV - http://dgv.tcag.ca)

In Patient 1, quality controls of the exome data revealed $97 \%$ of $10 x$ median coverage on target, with $98.03 \%$ of $\mathrm{Q}>30$; in Patient 2, 98\% of 10x median coverage on target, with $71.28 \%$ of $\mathrm{Q}>30$. It was identified six regions of homozygosity in Patient 2 using exome data (Supporting Information Table S1 and Supporting Information Figure S2), result in accordance with the reported parental consanguinity.

An active evaluation of a set of known genes for hepatoblastoma and Hirschsprung disease (Supporting Information Table S2) was performed by visual analysis of the BAM files to confirm that the exonic sequences of these genes were properly covered. Considering the data of both patients, a total of 333 rare coding nonsynonymous variants were detected, mapping to 317 genes.

No homozygous variant was detected in Patient 1 after filtering. A total of 72 rare heterozygous variants fulfilled the analysis criteria (seeMethodology), 24 of them were inherited from her mother, also heterozygous for these variants. Eleven out of the 72 variants were LoF affecting the genes C5orf47, CWC22, FRMPD2, GGCT, HAO2, KIR2DL4,LHX8, P2RX6, SPAG8, TMPRSS7, and ZNF215. Particularly, missense variants in the cancer predisposition genes $E R C C 2$ (c.545C $>\mathrm{T}$ rs142936491) and $H R A S$ (c.75G $>\mathrm{A}$ rs142218590) were identified, and one variant related to Hirschsprung disease in the ALDH1A2(c.1100A > T) gene.

After filtering, 261 rare variants were prioritized for further analysis in Patient 2, 243 of them in heterozygosity, comprising thirteen LoF affecting the genes APOB/PLCD4, CEP57L1, CNBD2, DENND6A, ELMOD3, FRG1B, GCA, FAM3A, LSM14A, PCDHGA1, RFPL1, SBK2, and SLC19A1; and 18 variants in homozygosity, including one LoF in the geneFAM3A. Fifty-six variants were also present in heterozygosity in the father. Variants were detected in the cancer-predisposing genes $A P C$ (c.3895G $>\mathrm{C}-\mathrm{rs} 1801166)$, $M C C(\mathrm{c} .862 \mathrm{G}>\mathrm{T}-\mathrm{rs} 34696815), O D C 1$ (rs116522452 - c.568G $>\mathrm{A}), B R C A 1$ (c.4039A $>\mathrm{G}-\mathrm{rs} 28897689)$, and ERBB2 (c.170A $>\mathrm{G}-\mathrm{rs} 140441229)$. Eight variants were mapped in genes related to cataract (ABCA4, COL4A4, HGSNAT, PCDH15, RPGRIP1, SLC16A12, SON, and VAT1 ), another clinical condition of the Patient 2. Details of the missense variants classified as damaging for at least five algorithms and the detected LoF variants are shown in Supporting Information Table S3 (57 for Patient 1 and 62 for Patient 2).

The list of 317 genes with rare coding non-synonymous variants from both patients was submitted to the prioritization tool VarElect ${ }^{33}$ using the phenotypes hepatoblastoma and congenital megacolon. This analysis revealed one gene directly related to Hirschsprung disease (ALDH1A2), and seventeen genes directly or indirectly (related with liver cancer, liver functions/structure, cancer, or syndromes that predispose to hepatoblastoma development) associated with hepatoblastoma (APC, BRCA1, CEP164, CYP1A1, ERBB2, ERCC2, FASN, HGS, HRAS, KMT2D, ODC1, PLCD4, SERPINA6, SLC25A47, SLC6A6, MCC, and ZNF215 ). Table 1 presents the germline VUS associated with the specific phenotypes of each patient.

Different CYP1A1 variants were identified in Patients 1 (c.1390C $>$ A) and 2 (c.877C $>$ G), both classified as VUS; additionally, one variant was detected in the Patient 2 affecting CEP164 (c.1429C>T), somatic mutations in this gene were previously reported in HB samples ${ }^{14}$. The variants in the CYP1A1 and CEP164 genes were validated by Sanger sequencing (Figure 2).

To explore the pathways in which the genes with rare germline coding non-synonymous variants are involved and their biological roles, we used Reactome Pathway Database (https://reactome.org/ - Version 71 Released). The gene set was enriched for pathways related to cancer, FGFR proteins family, Wnt signaling pathway metabolism, cytokine signaling in the immune system, post-translational protein modification, and developmental biology.

Somatic mutations in FFPE hepatoblastoma sample from Patient 1

A total of 36 somatic coding non-synonymous mutations were disclosed in the tumor sample, which mapped to 36 different genes, comprising two LoF, in the ANKRD22and FRY genes, and thirteen missense variants reported as pathogenic in more than five pathogenicity databases. Two of the detected mutations were 
already reported in COSMIC: CTNNB1(c.101G > T - COSM5670), variant already reported in hepatoblastomas, and RHBDL1 (c.1211G>A - COSM97348), detected in endometrium tumors.

Exome data was used to generate a genomic CNA profile from the tumor sample (Supporting Information Figure S3), only alterations larger than $3 \mathrm{Mb}$ were considered. Gains were detected in 2q, 8 and $17 \mathrm{q}$ in hepatoblastoma from Patient 1 (Supporting Information Table S4).

\section{DISCUSSION}

The association between congenital anomalies and pediatric cancer has long been recognized ${ }^{39}$. Recent studies evidenced an increased risk of pediatric cancer also in birth defects unrelated to chromosomal abnormalities or known genetic syndromes ${ }^{40,41}$. Hepatoblastomas, in particular, occur in association with a wide variety of congenital abnormalities ${ }^{8,42,43}$, especially craniosynostosis and renal anomalies ${ }^{12}$.

We report here two syndromic patients who developed the rare tumor hepatoblastoma, in addition to Hirschsprung disease, besides other anomalies, such as developmental delay, congenital cataract and nail dysplasia. The only clinical report of an association between hepatoblastoma and Hirschsprung disease corresponds to the Patient 2 of this study ${ }^{20}$.

Hirschsprung disease is a multifactorial condition with variable penetrance and expressivity, with several genes already known to cause this pathology when mutated ${ }^{22,23}$, however, germline mutations account for only $50 \%$ of the investigated cases of Hirschsprung disease ${ }^{44}$, pointing to the existence of yet unknown genetic mutations and underlying mechanisms contributing to the disease etiology. The gene set with rare coding germline variants identified in these two patients was enriched for pathways related to cancer, FGFR proteins family, Wnt signaling pathway metabolism, cytokine signaling in the immune system, post-translational protein modification, and developmental biology, suggesting they could have a broader role in cancer and congenital abnormalities.

Duplication in segment 14q23.2 have been documented in a few individuals in the control population and was classified as VUS. SYNE2, encompassed in the germline duplication at $14 \mathrm{q} 23.2$ detected in Patient 2, is involved in nuclear migration in retinal photoreceptor progenitors and is required for centrosome migration to the apical cell surface during early ciliogenesis (primary cilia formation). Alterations in $Y$ Y E2 have also been associated with retinal defects, the gene has an important role for proper retinal development ${ }^{45,46}$. This evidence could indicate that alterations in this gene might contribute to patient's congenital bilateral cataract and sensorineural deafness phenotypes.

In Patient 1, rare germline missense variants were identified in the cancer predisposition genes ERCC2 $(\mathrm{c} .545 \mathrm{C}>\mathrm{T})$ and HRAS (c.75G $>\mathrm{A})$, both in heterozygosity. ERCC2is involved in gene transcription and helps repair damaged $\mathrm{DNA}^{47,48}$. Homozygous variants in this gene are associated with predisposition to childhood solid tumor ${ }^{49}$, while and other variants in this gene have been related to hypoplastic nails, a clinical characteristic of Patient 1. A variant of maternal inheritance was identified in HRAS, which has been associated with Costello syndrome (OMIM \#218040), a rare dominant syndrome which increases the risk of tumors development ${ }^{50}$. It is not known how HRAS mutations cause the other features of Costello syndrome besides cancer predisposition such as intellectual disability, distinctive facial features, and heart problems, but many of the signs and symptoms probably result from cell overgrowth and abnormal cell division ${ }^{51-53}$. Interestingly, a LoF was observed in the ZNF215, disruptions in this gene were proposed to play a role in the etiology of Beckwith-Wiedemann syndrome.

Germline data of the Patient 2 also revealed four VUS in the cancer-predisposing genes $A P C$, a gene related to $\mathrm{FAP}$, one of the genetic conditions that predisposes to $\mathrm{HB}{ }^{2,54,55} ; M C C$, a regulator of the Wnt signaling pathway $^{56}$ and a candidate tumor suppressor gene ${ }^{57-59}$; ODC1, and BRCA1. One VUS were also observed in $E R B B 2$, a gene already associated with the megacolon phenotype ${ }^{60,61}$. In addition, eight variants were observed in genes related to cataract (ABCA4, COL4A4, HGSNAT, PCDH15, RPGRIP1, SLC16A12, SON, VAT1 $)^{62-64}$, a clinical condition of this patient.

In this study, we disclosed some potential candidate genes for hepatoblastoma and Hirschsprung disease. 
Particularly, variants in genes $C E P 164$ and $C Y P 1 A 1$ were interesting because they were previously observed in an HB exome study of somatic mutations ${ }^{14}$. CEP164 encodes a protein responsible for the repair signaling when DNA damage occurs ${ }^{65}$. This gene is involved in the stability of the genome and was previously found to be somatically mutated in two hepatoblastomas ${ }^{14}$. A germline variant was identified in the CEP164 of the Patient 2. We also detected, in both patients, germline variants in the geneCYP1A1. One variant in this gene was previously reported in a case of congenital hepatoblastoma ${ }^{14}$. CYP1A1 encodes a protein member of the cytochrome P450 superfamily of enzymes ${ }^{66}$; the expression of this gene is transcriptionally regulated through the $\mathrm{AhR}$ receptor, which plays an important role as a mediator of the adaptive response to xenobiotics and also contributes to normal physiology and embryonic development ${ }^{67}$.

Interestingly, another cytochrome involved in Hirschsprung disease was disclosed in this study. The germline variant in ALDH1A2(c.1100A > T) is described in COSMIC and ICGC as a somatic mutation with a high score of pathogenicity. The product of this gene is an enzyme that catalyzes the synthesis of retinoic acid from retinaldehyde ${ }^{68}$. Retinoic acid, the active derivative of vitamin A, is a hormonal signaling molecule that functions in developing and adult tissues. Studies of a homologous mouse gene suggest that this enzyme and the cytochrome CYP26A1 concurrently establish local embryonic retinoic acid levels, which facilitate later organs development ${ }^{69}$.

Hirschsprung disease is a highly heterogeneous genetic condition, and many cases remain idiopathic even after extensive molecular investigation, indicating the existence of underlying genes or genetic mechanism not yet recognized. The disease should be diagnosed early in the neonatal period, the delay in diagnosis and treatment can lead to development failure and other health problems in a considerable proportion of infants 70. In the same way, a growing number of cancer susceptibility genes are being identified and information is available in some public databases. However, for rare pediatric tumors, such as hepatoblastoma, the number of cases and the lack of grouping clinical information, makes it difficult to identify and understand the physiopathology of a causal germline mutations. Our data pointed out novel candidate genes ( $A L D H 1 A 2$, CEP164, CYP1A1, and SYNE2 ) for Hirschsprung disease biology with a potential functional role in the association with HB tumorigenesis (Figure 3 illustrates the main findings of this study). These results contribute to delineate future studies to understand the biology of Hirschsprung disease and its association with other conditions, like hepatoblastoma.

\section{ACKNOWLEDGMENTS}

We thank the patients and their families who enrolled in this study.

\section{FUNDING}

The present study was supported by grants from FAPESP (CEPID - Human Genome and Stem Cell Research Center 2013/08028-1; 2018/21047-9; fellowships: 2018/05961-2, 2016/04785-0, 2019/17423-8) and CNPq (141625/2016-3). The funders had no roles in the study design, data collection, and analysis, decision to publish, or preparation of the manuscript.

\section{COMPLIANCE WITH ETHICAL COMMITTEE}

Samples were recovered from patients enrolled in two Brazilian institutions: Patient 1 was referred from Department of Pediatrics Hospital da Baleia - Brazil, and Patient 2 was referred from Hospital da Criança Conceição - Brazil. The Research Ethics Committee of the respective Institutions approved this research using these biological samples, and all samples were collected after informed signed consent was obtained from parents or legal guardians.

\section{CREDIT AUTHOR STATEMENT}

All authors contributed to the study conception and design. Material preparation, data collection and analysis were performed by Talita Aguiar, Anne Teixeira, Juliana Sobral, Isabela Cunha, Eugênia Valadares, Raquel Pinto, and Ana Krepischi. The first draft of the manuscript was written by Talita Aguiar, Anne Teixeira 
and Ana Krepischi and all authors commented on previous versions of the manuscript. All authors read and approved the final manuscript.

\section{CONFLICTS OF INTEREST}

We declare that we have no conflicts of interest.

\section{REFERENCES}

1. Saletta F, Dalla Pozza L, Byrne JA. Genetic causes of cancer predisposition in children and adolescents. Transl Pediatr . 2015;4(2):67-75. doi:10.3978/j.issn.2224-4336.2015.04.08

2. Zhang J, Walsh MF, Wu G, et al. Germline Mutations in Predisposition Genes in Pediatric Cancer. $N$ Engl J Med . 2015;373(24):2336-2346. doi:10.1056/NEJMoa1508054

3. Grobner SN, Worst BC, Weischenfeldt J, et al. The landscape of genomic alterations across childhood cancers. Nature . 2018;555(7696):321-327. doi:10.1038/nature25480

4. Ma X, Liu Y, Liu Y, et al. Pan-cancer genome and transcriptome analyses of 1,699 paediatric leukaemias and solid tumours. Nature . 2018. doi:10.1038/nature25795

5. Sweet-Cordero EA, Biegel JA. The genomic landscape of pediatric cancers: Implications for diagnosis and treatment. Science . 2019;363(6432):1170-1175. doi:10.1126/science.aaw3535

6. Agha MM, Williams JI, Marrett L, To T, Zipursky A, Dodds L. Congenital abnormalities and childhood cancer: A cohort record-linkage study. Cancer . 2005. doi:10.1002/cncr.20985

7. Merks JHM, Caron HN, Hennekam RCM. High incidence of malformation syndromes in a series of 1,073 children with cancer. Am J Med Genet A . 2005;134A(2):132-143. doi:10.1002/ajmg.a.30603

8. Scollon S, Anglin AK, Thomas M, Turner JT, Wolfe Schneider K. A Comprehensive Review of Pediatric Tumors and Associated Cancer Predisposition Syndromes. J Genet Couns . 2017;26(3):387-434. doi:10.1007/s10897-017-0077-8

9. Heck JE, Meyers TJ, Lombardi C, et al. Case-control study of birth characteristics and the risk of hepatoblastoma. Cancer Epidemiol . 2013. doi:10.1016/j.canep.2013.03.004

10. Stiller CA, Pritchard J, Steliarova-Foucher E. Liver cancer in European children: incidence and survival, 1978-1997. Report from the Automated Childhood Cancer Information System project. Eur J Cancer . 2006;42(13):2115-2123. doi:10.1016/j.ejca.2006.05.011

11. Czauderna P, Garnier H. Hepatoblastoma: current understanding, recent advances, and controversies. F1000Research . 2018;7:53. doi:10.12688/f1000research.12239.1

12. de Camargo B, de Oliveira Ferreira JM, de Souza Reis R, Ferman S, de Oliveira Santos M, Pombode-Oliveira MS. Socioeconomic status and the incidence of non-central nervous system childhood embryonic tumours in Brazil. BMC Cancer . 2011. doi:10.1186/1471-2407-11-160

13. Instituto Nacional de Cancer Jose Alencar Gomes da Silva (INCA).Incidencia, Mortalidade e Morbidade Hospitalar Por Cancer Em Criancas, Adolescentes e Adultos Jovens No Brasil: Informacoes Dos Registros de Cancer e Do Sistema de Mortalidade. (Coordenacao de Prevencao e Vigilancia, ed.). Rio de Janeiro: INCA; 2016.

14. Aguiar TFM, Rivas MP, Costa S, et al. Insights Into the Somatic Mutation Burden of Hepatoblastomas From Brazilian Patients. Front Oncol . 2020;10:556. doi:10.3389/fonc.2020.00556

15. DeBaun MR, Tucker MA. Risk of cancer during the first four years of life in children from The BeckwithWiedemann Syndrome Registry. J Pediatr . 1998;132(3 Pt 1):398-400. 
16. Kim SY, Jung S-H, Kim MS, et al. Genomic profiles of a hepatoblastoma from a patient with BeckwithWiedemann syndrome with uniparental disomy on chromosome $11 \mathrm{p} 15$ and germline mutation of APC and PALB2. Oncotarget . 2017. doi:10.18632/oncotarget.20515

17. Hirschman BA, Pollock BH, Tomlinson GE. The spectrum of APC mutations in children with hepatoblastoma from familial adenomatous polyposis kindreds. J Pediatr . 2005. doi:10.1016/j.jpeds.2005.04.019

18. Pereira EM, Marion R, Ramesh KH, Kim JS, Ewart M, Ricafort R. Hepatoblastoma in a mosaic trisomy 18 patient. J Pediatr Hematol Oncol . 2012;34(4):e145-8. doi:10.1097/MPH.0b013e3182459ee8

19. Turcotte LM, Georgieff MK, Ross JA, et al. Neonatal medical exposures and characteristics of low birth weight hepatoblastoma cases: a report from the Children's Oncology Group. Pediatr Blood Cancer . 2014;61(11):2018-2023. doi:10.1002/pbc.25128

20. Pinto RB, Ramos ARL, Backes AN, et al. Hirschsprung disease and hepatoblastoma: case report of a rare association. Sao Paulo Med J . 2016;134(2):171-175. doi:10.1590/1516-3180.2014.9200311

21. Best KE, Addor M-C, Arriola L, et al. Hirschsprung's disease prevalence in Europe: a register based study. Birth Defects Res A Clin Mol Teratol . 2014;100(9):695-702. doi:10.1002/bdra.23269

22. Heuckeroth RO. Hirschsprung disease - integrating basic science and clinical medicine to improve outcomes. Nat Rev Gastroenterol Hepatol . 2018;15(3):152-167. doi:10.1038/nrgastro.2017.149

23. Haricharan RN, Georgeson KE. Hirschsprung disease. Semin Pediatr Surg . 2008;17(4):266-275. doi:10.1053/j.sempedsurg.2008.07.005

24. Salehpour S, Hashemi-Gorji F, Soltani Z, Ghafouri-Fard S, Miryounesi M. Association of a Novel Nonsense Mutation in KIAA1279 with Goldberg-Shprintzen Syndrome. Iran J child Neurol . 2017;11(1):7074. http://www.ncbi.nlm.nih.gov/pubmed/28277559. Accessed August 26, 2019.

25. Badner JA, Sieber WK, Garver KL, Chakravarti A. A genetic study of Hirschsprung disease. Am J Hum Genet . 1990;46(3):568-580.

26. Amiel J, Sproat-Emison E, Garcia-Barcelo M, et al. Hirschsprung disease, associated syndromes and genetics: a review. J Med Genet . 2008;45(1):1-14. doi:10.1136/jmg.2007.053959

27. Camilleri M, Wieben E, Eckert D, et al. Familial chronic megacolon presenting in childhood or adulthood: Seeking the presumed gene association. Neurogastroenterol Motil . 2019;31(4):e13550. doi:10.1111/nmo.13550

28. Sambrook J, Russell DW. Purification of nucleic acids by extraction with phenol:chloroform. CSH Protoc . 2006;2006(1). doi:10.1101/pdb.prot4455

29. Campos AHJFM, Silva AA, Mota LDDC, et al. The value of a tumor bank in the development of cancer research in Brazil: 13 years of experience at the a C camargo hospital. Biopreserv Biobank . 2012;10(2):168173. doi:10.1089/bio.2011.0032

30. Li H. Aligning Sequence Reads, Clone Sequences and Assembly Contigs with BWA-MEM .; 2013. http://github.com/lh3/bwa. Accessed May 18, 2020.

31. McKenna A, Hanna M, Banks E, et al. The genome analysis toolkit: A MapReduce framework for analyzing next-generation DNA sequencing data. Genome Res . 2010;20(9):1297-1303. doi:10.1101/gr.107524.110

32. Challis D, Yu J, Evani US, et al. An integrative variant analysis suite for whole exome next-generation sequencing data. BMC Bioinformatics . 2012;13(1). doi:10.1186/1471-2105-13-8

33. Stelzer G, Plaschkes I, Oz-Levi D, et al. VarElect: The phenotype-based variation prioritizer of the GeneCards Suite. BMC Genomics . 2016. doi:10.1186/s12864-016-2722-2 
34. Kohler S, Carmody L, Vasilevsky N, et al. Expansion of the Human Phenotype Ontology (HPO) knowledge base and resources. Nucleic Acids Res . 2019. doi:10.1093/nar/gky1105

35. Hampel H, Bennett RL, Buchanan A, Pearlman R, Wiesner GL, Guideline Development Group, American College of Medical Genetics and Genomics Professional Practice and Guidelines Committee and National Society of Genetic Counselors Practice Guidelines Committee. A practice guideline from the American College of Medical Genetics and Genomics and the National Society of Genetic Counselors: referral indications for cancer predisposition assessment. Genet Med . 2015;17(1):70-87. doi:10.1038/gim.2014.147

36. LSue Richards, PhD1, Nazneen Aziz, PhD2, 16 , Sherri Bale, PhD3, David Bick M, , Soma Das P, Julie Gastier-Foster, PhD6, 7, 8, Wayne W. Grody, MD, PhD9, 10, 11, Madhuri Hegde P, et al. Standards and Guidelines for the Interpretation of Sequence Variants: A Joint Consensus Recommendation of the American College of Medical Genetics and Genomics and the Association for Molecular Pathology Sue. Genet Med . 2015. doi:10.1038/gim.2015.30.Standards

37. Kopanos C, Tsiolkas V, Kouris A, et al. VarSome: the human genomic variant search engine. Bioinformatics . 2019. doi:10.1093/bioinformatics/bty897

38. Oliveira D, Leal GF, Sertie AL, et al. 10q23.31 microduplication encompassing PTEN decreases mTOR signalling activity and is associated with autosomal dominant primary microcephaly. J Med Genet . October 2018. doi:10.1136/jmedgenet-2018-105471

39. Agha MM, Williams JI, Marrett L, To T, Zipursky A, Dodds L. Congenital abnormalities and childhood cancer. Cancer . 2005;103(9):1939-1948. doi:10.1002/cncr.20985

40. Siegel RL, Miller KD, Jemal A. Cancer Statistics, 2017. CA Cancer J Clin . 2017;67(1):7-30. doi: $10.3322 /$ caac. 21387

41. Norwood MS, Lupo PJ, Chow EJ, et al. Childhood cancer risk in those with chromosomal and nonchromosomal congenital anomalies in Washington State: 1984-2013. PLoS One . 2017;12(6):e0179006. doi:10.1371/journal.pone.0179006

42. Ansell P, Mitchell CD, Roman E, Simpson J, Birch JM, Eden TOB. Relationships between perinatal and maternal characteristics and hepatoblastoma: a report from the UKCCS. Eur J Cancer . 2005;41(5):741-748. doi:10.1016/j.ejca.2004.10.024

43. Narod SA, Hawkins MM, Robertson CM, Stiller CA. Congenital anomalies and childhood cancer in Great Britain. Am J Hum Genet . 1997;60(3):474-485.

44. Butler Tjaden NE, Trainor PA. The developmental etiology and pathogenesis of Hirschsprung disease. Transl Res . 2013;162(1):1-15. doi:10.1016/j.trsl.2013.03.001

45. Maddox DM, Collin GB, Ikeda A, et al. A Mutation in Syne2 Causes Early Retinal Defects in Photoreceptors, Secondary Neurons, and Muller Glia. Invest Ophthalmol Vis Sci . 2015;56(6):3776-3787. doi:10.1167/iovs.14-16047

46. Falk N, Kessler K, Schramm S-F, et al. Functional analyses of Pericentrin and Syne-2 interaction in ciliogenesis. J Cell Sci . 2018;131(16). doi:10.1242/jcs.218487

47. Takayama K, Salazar EP, Lehmann A, Stefanini M, Thompson LH, Weber CA. Defects in the DNA repair and transcription gene ERCC2 in the cancer-prone disorder xeroderma pigmentosum group D. Cancer Res . 1995;55(23):5656-5663. http://www.ncbi.nlm.nih.gov/pubmed/7585650. Accessed August 6, 2019.

48. Liu H, Rudolf J, Johnson KA, et al. Structure of the DNA repair helicase XPD. Cell . 2008;133(5):801812. doi:10.1016/j.cell.2008.04.029

49. Lehmann AR, McGibbon D, Stefanini M. Xeroderma pigmentosum.Orphanet J Rare Dis . 2011. doi:10.1186/1750-1172-6-70 
50. Aoki Y, Niihori T, Kawame H, et al. Germline mutations in HRAS proto-oncogene cause Costello syndrome. Nat Genet . 2005;37(10):1038-1040. doi:10.1038/ng1641

51. Aoki Y, Matsubara Y. Ras/MAPK syndromes and childhood hemato-oncological diseases. Int J Hematol . 2013;97(1):30-36. doi:10.1007/s12185-012-1239-y

52. Gripp KW, Innes AM, Axelrad ME, et al. Costello syndrome associated with novel germline HRAS mutations: An attenuated phenotype? Am J Med Genet Part A . 2008. doi:10.1002/ajmg.a.32227

53. Chiu ATG, Leung GKC, Chu YWY, Gripp KW, Chung BHY. A novel patient with an attenuated Costello syndrome phenotype due to an HRAS mutation affecting codon 146-Literature review and update. Am J Med Genet Part A . 2017. doi:10.1002/ajmg.a.38118

54. Hughes LJ, Michels V V. Risk of hepatoblastoma in familial adenomatous polyposis. Am J Med Genet . 1992;43(6):1023-1025. doi:10.1002/ajmg.1320430621

55. Hirschman BA, Pollock BH, Tomlinson GE. The spectrum of APC mutations in children with hepatoblastoma from familial adenomatous polyposis kindreds. J Pediatr . 2005;147(2):263-266. doi:10.1016/j.jpeds.2005.04.019

56. Pangon L, Mladenova D, Watkins L, et al. MCC inhibits beta-catenin transcriptional activity by sequestering DBC1 in the cytoplasm.Int J Cancer . 2015;136(1):55-64. doi:10.1002/ijc.28967

57. Fukuyama R, Niculaita R, Ng KP, et al. Mutated in colorectal cancer, a putative tumor suppressor for serrated colorectal cancer, selectively represses $\beta$-catenin-dependent transcription. Oncogene . 2008;27(46):6044-6055. doi:10.1038/onc.2008.204

58. Kinzler KW, Vogelstein B. Cancer-susceptibility genes. Gatekeepers and caretakers. Nature . 1997;386(6627):761,763. doi:10.1038/386761a0

59. Currey N, Jahan Z, Caldon CE, et al. Mouse Model of Mutated in Colorectal Cancer Gene Deletion Reveals Novel Pathways in Inflammation and Cancer. CMGH . 2019;7(4):819-839. doi:10.1016/j.jcmgh.2019.01.009

60. Erwin CR, Warner BW. Hirschsprung lost his nerve.Gastroenterology . 2003;125(6):1900-1902. doi:10.1053/j.gastro.2003.07.019

61. Pectasides E, Bass AJ. ERBB2 emerges as a new target for colorectal cancer. Cancer Discov . 2015;5(8):799-801. doi:10.1158/2159-8290.CD-15-0730

62. Shiels A, Hejtmancik JF. Genetics of human cataract. Clin Genet . 2013;84(2):120-127. doi:10.1111/cge.12182

63. Zhai Y, Li J, Yu W, et al. Targeted Exome Sequencing of Congenital Cataracts Related Genes: Broadening the Mutation Spectrum and Genotype-Phenotype Correlations in 27 Chinese Han Families. Sci Rep . 2017;7(1):1-9. doi:10.1038/s41598-017-01182-9

64. Francis PJ, Berry V, Bhattacharya SS, Moore AT. The genetics of childhood cataract. J Med Genet . 2000;37(7):481-488. doi:10.1136/jmg.37.7.481

65. Sivasubramaniam S, Sun X, Pan Y-R, Wang S, Lee EY-HP. Cep164 is a mediator protein required for the maintenance of genomic stability through modulation of MDC1, RPA, and CHK1. Genes Dev . 2008;22(5):587-600. doi:10.1101/gad.1627708

66. Stejskalova L, Pavek P. The function of cytochrome P450 1A1 enzyme (CYP1A1) and aryl hydrocarbon receptor (AhR) in the placenta. Curr Pharm Biotechnol . 2011;12(5):715-730.

67. Delescluse C, Lemaire G, de Sousa G, Rahmani R. Is CYP1A1 induction always related to AHR signaling pathway? Toxicology . 2000;153(1-3):73-82. doi:10.1016/s0300-483x(00)00305-x 
68. Manolescu DC, El-Kares R, Lakhal-Chaieb L, Montpetit A, Bhat P V, Goodyer P. Newborn serum retinoic acid level is associated with variants of genes in the retinol metabolism pathway. Pediatr Res . 2010;67(6):598-602. doi:10.1203/PDR.0b013e3181dcf18a

69. Ricard MJ, Gudas LJ. Cytochrome P450 Cyp26a1 alters spinal motor neuron subtype identity in differentiating embryonic stem cells. J Biol Chem . 2013;288(40):28801-28813. doi:10.1074/jbc.M113.474254

70. Martucciello G. Hirschsprung's disease, one of the most difficult diagnoses in pediatric surgery: A review of the problems from clinical practice to the bench. Eur J Pediatr Surg . 2008;18(3):140-149. doi:10.1055/s2008-1038625

\section{LEGENDS OF FIGURES}

Figure 1. Rare germline interrupted duplication identified at 14q23.2 in Patient 2. The Y axis shows the copy number values in $\log 2$ and the $\mathrm{X}$ axis shows probes plotted according to genomic coordinates. The duplicated regions are highlighted in purple in the graph, with positive log2 values. Blue bars (top of the figure) show the localization of the duplication at $14 \mathrm{q} 23.2$.

Figure 2. Germline missense variants detected by exome analysis in Patients 1 and 2 and validated by Sanger Sequencing. Different rare variants were identified in the CYP1A1 gene in A. Patient 1 (c.1390C >A) and B. Patient 2 (c.877C > G); C. CEP164 variant detected in Patient 2 (c.1429C>T).

Figure 3. Main observations. Specific phenotypes and associated genes presenting rare germline coding non-synonymous variants in Patients 1 and 2. Hepatoblastoma and Hirschsprung disease are shared phenotypes between the patients, and $C Y P 1 A 1$ is the only gene with rare variants detected in both patients. The phenotype and related gene are linked by color. The genes associated to more than one phenotype are marked with an asterisk of the color of the second associated phenotype.

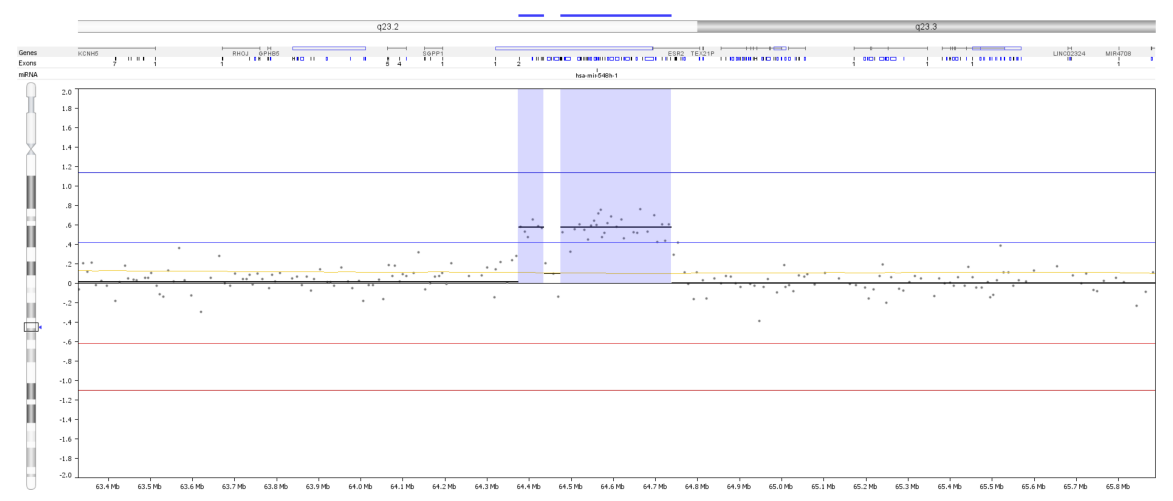


A. Patient 1: CYP1A1

RelSeq Genes 105 Interim V1, NCBI

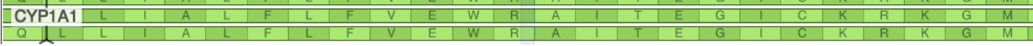

Patient 1

Coverage

要 40 ITTGCAGCAGGATAGCCAGGAGAGAAAGACCTCCCAGCGGGCAATGGTCTCACCGATACACTTCCGCTTGCCCATS

Pile-up

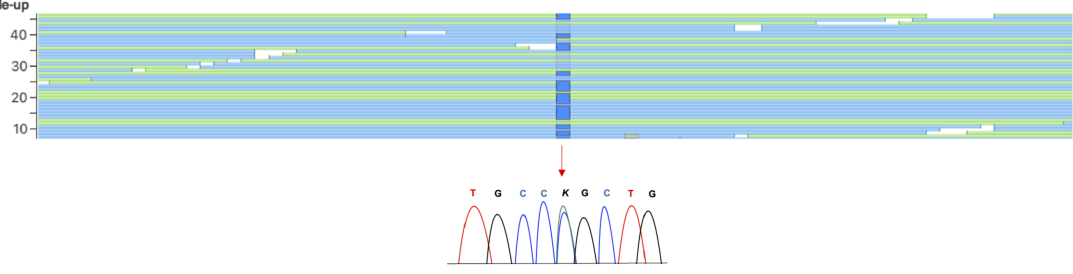

B. Patient 2: CYP1A1

RefSeq Genes 105 Interim v1, NCBI

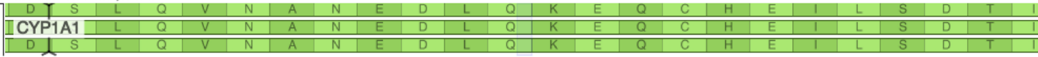

Patient 2

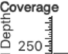

A 100 ATCTGACAGCTGGACATTGGCGTTCTCATCCAGCTGCTTCTCCTGACAGTGCTCAATCAGGCTGTCTGTGA

Pile-up
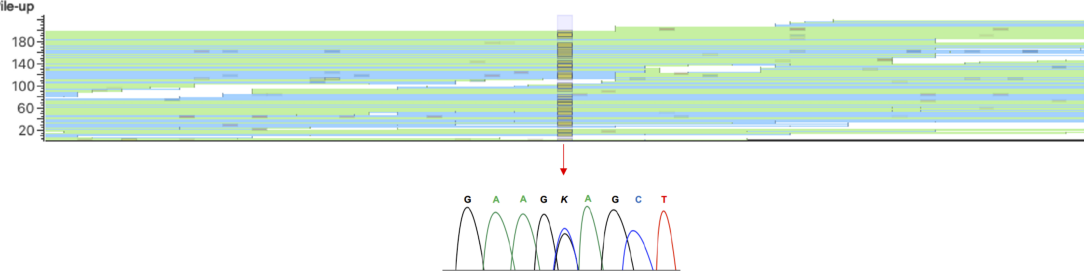

C. Patient 2: CEP164

RefSeq Genes 105 Interim v1, NCBI

CEP164

\begin{tabular}{|ll|l|l|l|l|l|l|l|l|l|l|l|l|l|l|} 
D & F & G & F & R & S & R & I & S & E & H & L & L & D & V & D \\
\hline \hline D & F & G & F & R & S & R & I & S & E & H & L & L & D & V & D \\
\hline
\end{tabular}

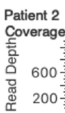

200 DTTTGCATCTCCACTGTCTCCAGGACTTCGGTTTTRGCAGCCGGATCTCGGAGCACCTGCTGGATGTTG

Pile-up

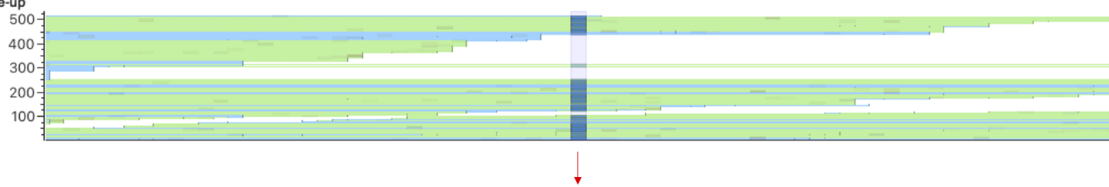

mMmm 


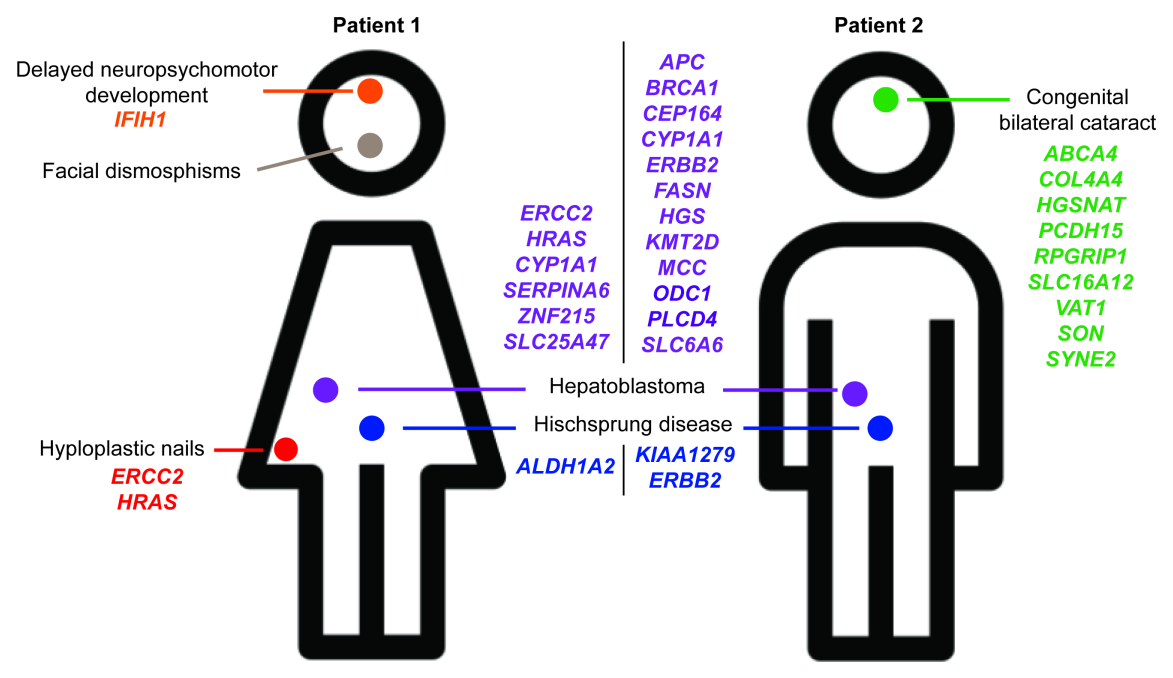

\section{Hosted file}

Table 1.docx available at https://authorea.com/users/328345/articles/464307-birthdefects-and-childhood-cancer-a-shared-biological-pathway-for-hirschsprung-disease-andhepatoblastoma-development 